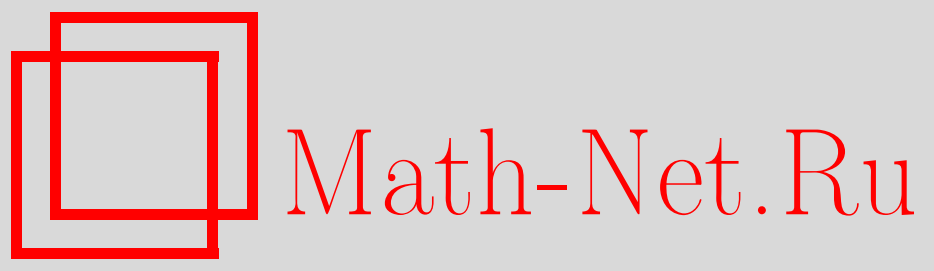

V. I. Bernik, N. Budarina, H. O'Donnell, On Regular Systems of Real Algebraic Numbers of Third Degree in Short Intervals, Совр. пробл. матем., 2013, выпуск 17, 61-75

DOI: https://doi.org/10.4213/spm43

Использование Общероссийского математического портала Math-Net.Ru подразумевает, что вы прочитали и согласны с пользовательским соглашением http://www . mathnet.ru/rus/agreement

Параметры загрузки:

IP : 54.84 .234 .179

26 апреля 2023 г., 16:18:21

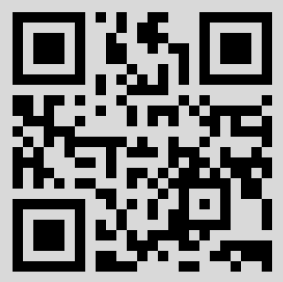




\title{
On Regular Systems of Real Algebraic Numbers of Third Degree in Short Intervals
}

\author{
V.I. Bernik ${ }^{a}$, N. Budarina ${ }^{b c}, H$. O'Donnell $^{b}$ \\ ${ }^{a}$ Institute of Mathematics, National Academy of Sciences of Belarus \\ ${ }^{b}$ Dublin Institute of Technology, Ireland \\ ${ }^{c}$ Institute for Applied Mathematics, \\ Khabarovsk Division, Far-Eastern Branch of the Russian Academy of Sciences
}

In memory of Anatolii Karatsuba

About Anatolii Karatsuba. Anatolii Alexeevich Karatsuba was always closely connected to Belarus. Many of his fellow mathematicians from the North Caucasus had found employment in this country. He went mountain climbing together with many Belarusians, and always had high regard for their professional and personal qualities. Anatolii Karatsuba was a peer and a close friend to the founder of the Belarusian number-theoretic school academician Vladimir Gennadievich Sprindžuk. They both had immense respect for each other's opinions.

The first two authors had numerous encounters with Anatolii Karatsuba at various mathematical conferences. Our conversations, although mostly brief, were very informative. We discussed the most recent mathematical results, sports, mountain climbing and hiking, the latest news from Belarus. The conversations were always constructive: when asked for assistance in the matter of organizing a conference, supporting an applicant for the membership in the Academy of Sciences or some Belarusian mathematical award he always pledged his support and signed petitions to various official institutions of the Soviet Union and the Republic of Belarus.

Every Belarusian researcher that knew Prof. Karatsuba immensely respected him as both a mathematician and a person.

\section{Introduction}

In [1] Baker and Schmidt introduced the definition of regular systems and proved the regularity of real algebraic numbers of any degree. This gives the possibility of obtaining the lower bound for the Hausdorff dimension of the set of real numbers which are approximated by algebraic numbers with a given order of approximation. The result of Baker and Schmidt was improved by Bernik [2]. Beresnevich [3] proved the regularity of real algebraic numbers and his result was the best possible for functions defined on the set of algebraic numbers. The latter improvement enabled him to prove the divergence case of a Khinchine type theorem for polynomials of any degree.

The Lebesgue measure of a measurable set $S \subset \mathbb{R}$ is denoted by $|S|$. The set of real algebraic numbers of degree $n$ is denoted by $A_{n}$. Let $c_{i}$ denote a positive constant.

Definition 1. Let $\Gamma$ be a countable set of real numbers and $N: \Gamma \rightarrow \mathbb{R}$ be a positive function. The pair $(\Gamma, N)$ is called a regular system if there exists a constant $c_{1}=c_{1}(\Gamma, N)>0$ such that for any interval $I \subset \mathbb{R}$ there exists a sufficiently large number $T_{0}=T_{0}(\Gamma, N, I)>0$ such that for any integer $T>T_{0}$ there exist $\gamma_{1}, \gamma_{2}, \ldots, \gamma_{t}$ in $\Gamma \cap I$ such that

$$
\begin{gathered}
N\left(\gamma_{i}\right) \leqslant T, \quad 1 \leqslant i \leqslant t \\
\left|\gamma_{i}-\gamma_{j}\right|>T^{-1}, \quad 1 \leqslant i<j \leqslant t, \\
t>c_{1}|I| T .
\end{gathered}
$$


It is not difficult to show that the set of rational numbers $p / q, \operatorname{gcd}(p, q)=1$, with the function $N(p / q)=q^{2}$ is a regular system. In the above results of Baker and Schmidt, Bernik, and Beresnevich, it was shown that the set of real algebraic numbers $\alpha$ of degree $\leqslant n$ together with the function $N(\alpha)=H(\alpha)^{n+1} \log ^{-v} H(\alpha)$ forms a regular system for $v=3 n(n+1), 2$ and 0 respectively. Here $H(\alpha)$ is the height of algebraic number $\alpha$, i.e. the maximum of the absolute values of the coefficients of the minimal polynomial of $\alpha$. In [3] the constant $c_{1}$ is calculated, but $T_{0}$ is not (both of these are in the definition of a regular system).

In [4] it is shown that for a given finite interval $I$ in $[0,1]$ the value of $T_{0}$ is equal to

$$
T_{0}(\mathbb{Q}, N, I)=10^{4}|I|^{-2} \log ^{2}\left(100|I|^{-1}\right)
$$

for $n=1$, and in [5] that

$$
T_{0}\left(A_{2}, N, I\right)=72^{3}|I|^{-3} \log ^{3}\left(72|I|^{-1}\right)
$$

for $n=2$, where $\mathbb{Q}$ is the set of rational numbers. In [4] Bugeaud notes that for $n \geqslant 3$ the relation between $|I|$ and $T_{0}$ is not presently known.

In this paper for $n=3$ the relationship between $|I|$ and $T_{0}$ is examined, and it is shown that $T_{0}\left(A_{3}, N, I\right)=c_{2}|I|^{-4}$. Probably such type of the relation is preserved for any $n$, i.e. $T_{0}\left(A_{n}, N, I\right)=$ $c_{3}|I|^{-(n+1)}$.

Given a polynomial $P(x)=a_{n} x^{n}+a_{n-1} x^{n-1}+\cdots+a_{1} x+a_{0} \in \mathbb{Z}[x]$ with $a_{n} \neq 0, \operatorname{deg} P=n$ is the degree of $P$ and $H(P)=\max _{0 \leqslant i \leqslant n}\left|a_{i}\right|$ is the height of $P$. For a positive integer $Q$ define the set of polynomials

$$
\mathcal{P}_{3}^{\prime}(Q)=\{P \in \mathbb{Z}[x]: \operatorname{deg} P=3, H(P) \leqslant Q\} .
$$

\section{Statement of results}

TheOREM 1. Let I be a finite interval contained in $[-1 / 2,1 / 2]$. Then there exist positive constants $c_{1}, c_{4}$ and a positive number $T_{0}=c_{4}|I|^{-4}$ such that for any $T \geqslant T_{0}$ there exist numbers $\alpha_{1}, \ldots, \alpha_{t} \in A_{3} \cap I$ such that

$$
\begin{gathered}
H\left(\alpha_{i}\right) \leqslant T^{1 / 4}, \quad 1 \leqslant i \leqslant t \\
\left|\alpha_{i}-\alpha_{j}\right| \geqslant T^{-1}, \quad 1 \leqslant i<j \leqslant t, \\
t \geqslant c_{1} T|I| .
\end{gathered}
$$

Note that from Theorem 1 it follows that the set of real algebraic numbers $\alpha$ of degree 3 , together with the function $N(\alpha)=H^{4}(\alpha)$ form a regular system on $[-1 / 2,1 / 2]$.

Let $\delta_{0} \in \mathbb{R}^{+}$. Denote by $\overline{\mathcal{L}}_{3}=\overline{\mathcal{L}}_{3}\left(Q, \delta_{0}, I\right)$ the set of $x \in I$, for which the system of inequalities

$$
|P(x)|<Q^{-3}, \quad\left|P^{\prime}(x)\right|<\delta_{0} Q
$$

are satisfied for some $P \in \mathcal{P}_{3}^{\prime}(Q)$. The proof of theorem 1 is based on the following metric result.

THEOREM 2. For any real number $s$, where $0<s<1$, there exists a constant $\delta_{0}$, which satisfies the following property. For any interval $I \subset[-1 / 2,1 / 2]$ there exists a sufficiently large number $Q_{0}=Q_{0}(I)$ and a constant $c_{5}$ independent of $Q_{0}$ such that

$$
|I|>c_{5} Q_{0}^{-1}
$$

and for all $Q>Q_{0}$

$$
\left|\overline{\mathcal{L}}_{3}\left(Q, \delta_{0}, I\right)\right|<s|I| .
$$

REMARK 1 . Let $J$ be any interval of length $\leqslant 1$ and $J_{0}$ be a finite interval contained in $J$. There exists an integer $t$ such that $J_{0, t}=\left(J_{0}+t\right) \cap[-1 / 2,1 / 2]$ has measure at most $\left|J_{0}\right| / 2$. Then we pass from the polynomial $P$ to the polynomial $P_{1}$ in the system $(3)$, where $P_{1}(x)=P(x-t)$ with 
$H\left(P_{1}\right)<c(t) H(P) \leqslant c(t) Q$ and $x \in J_{0, t}$. Therefore, we can reduce the problem from an arbitrary interval of length $\leqslant 1$ to the similar problem in interval $[-1 / 2,1 / 2]$ only with more complicated connection between $H\left(P_{1}\right)$ and $Q$.

REMARK 2. Throughout the proof we use the fact that some of the statements are true for sufficiently large $Q$, and $Q$ is greater than some value $c(n)|I|^{-1}$. Denote by $c_{5}$ in Theorem 2 such a value $c_{5}=c_{5}(n)$ that all the statements will be true.

The proof of Theorem 2 uses the concept of essential and inessential domains extensively. This concept was first introduced by Sprindžuk in [6] and is described here.

DEFINITION 2. Let $\mathcal{P}$ be a set of polynomials satisfying certain conditions and $\sigma(P)$ be a set of points (defined for each $P \in \mathcal{P}$ ) which meet certain conditions. A domain $\sigma(P)$ is called essential if

$$
\left|\sigma(P) \cap \bigcup_{Q \in \mathcal{P}} \sigma(Q)\right|<\frac{1}{2}|\sigma(P)|
$$

and is called inessential otherwise.

\section{Proof of Theorem 2}

Let $I$ be a finite interval contained in $[-1 / 2,1 / 2]$. The proof of the theorem is in two parts: the first when $\left|P^{\prime}(x)\right| \gg Q^{-1}$ and the second when $\left|P^{\prime}(x)\right| \ll Q^{-1}$. The first part has five cases.

Case 1. Define $\widetilde{\mathcal{L}}_{3}$ as the subset of $\overline{\mathcal{L}}_{3}$ containing the set of points $x \in I$, for which there exists a polynomial $P \in \mathcal{P}^{\prime}{ }_{3}(Q)$ such that the system

$$
|P(x)|<Q^{-3}, \quad 2^{6} Q^{-1}<\left|P^{\prime}(x)\right|<\delta_{0} Q
$$

holds.

Denote by $\sigma_{0}(P)$ the set of solutions $x \in I$ of $(5)$ for a fixed polynomial $P \in \mathcal{P}_{3}^{\prime}(Q)$. Then $\widetilde{\mathcal{L}}_{3}$ can be written as $\widetilde{\mathcal{L}}_{3}=\bigcup_{P \in \mathcal{P}_{3}^{\prime}(Q)} \sigma_{0}(P)$. Let $\alpha_{1}, \alpha_{2}$ and $\alpha_{3}$ be the roots of $P \in \mathcal{P}_{3}^{\prime}(Q)$ in $\mathbb{C}$. Define the sets

$$
S_{P}\left(\alpha_{i}\right)=\left\{x \in \mathbb{R}:\left|x-\alpha_{i}\right|=\min _{1 \leqslant j \leqslant 3}\left|x-\alpha_{j}\right|\right\}, \quad i=1,2,3 .
$$

Lemma 1. Let $P \in \mathcal{P}_{3}^{\prime}$ and $x \in S\left(\alpha_{i}\right)$. Then

$$
\begin{array}{ll}
\left|x-\alpha_{i}\right| \leqslant 3|P(x)|\left|P^{\prime}(x)\right|^{-1} & \text { for } \quad P^{\prime}(x) \neq 0, \\
\left|x-\alpha_{i}\right| \leqslant 4|P(x)|\left|P^{\prime}\left(\alpha_{i}\right)\right|^{-1} & \text { for } \quad P^{\prime}\left(\alpha_{i}\right) \neq 0 .
\end{array}
$$

Lemma 1 is proven in [2] and [6] respectively.

For simplicity only $S_{P}\left(\alpha_{1}\right) \cap I$, is considered as the arguments are the same for the other two $S_{P}\left(\alpha_{i}\right) \cap I$. Let $x \in \sigma_{0}(P) \cap S_{P}\left(\alpha_{1}\right)$. Then it is not difficult to verify that $\left|\alpha_{1}\right|<Q^{-1}+1 / 2 \leqslant 1$ for $Q \geqslant 2$. Thus, $\left|P^{(k)}\left(\alpha_{1}\right)\right| \leqslant 8 Q$ for $k=1,2,3$ and $Q \geqslant 2$. By Taylor's formula

$$
P^{\prime}(x)=P^{\prime}\left(\alpha_{1}\right)+P^{\prime \prime}\left(\alpha_{1}\right)\left(x-\alpha_{1}\right)+P^{\prime \prime \prime}\left(\alpha_{1}\right) \frac{\left(x-\alpha_{1}\right)^{2}}{2} .
$$

Estimating the second and the third terms by using Lemma 1 gives:

$$
\begin{aligned}
\left|P^{\prime \prime}\left(\alpha_{1}\right)\left(x-\alpha_{1}\right)\right| & \leqslant 8 Q 3 Q^{-3}\left|P^{\prime}(x)\right|^{-1}<\frac{3}{8} Q^{-1} \\
\left|P^{\prime \prime \prime}\left(\alpha_{1}\right) \frac{\left(x-\alpha_{1}\right)^{2}}{2}\right| & \leqslant 4 Q\left(3 Q^{-3}\left|P^{\prime}(x)\right|^{-1}\right)^{2}<3^{2} \cdot 2^{-10} Q^{-3}<\frac{1}{8} Q^{-1}
\end{aligned}
$$


for $\left|P^{\prime}(x)\right|>2{ }^{6} Q^{-1}$ and $Q \geqslant 2$. It follows from (5), (6) and (7) that

$$
\frac{1}{2}\left|P^{\prime}(x)\right|<\left|P^{\prime}\left(\alpha_{1}\right)\right|<2\left|P^{\prime}(x)\right| \text {. }
$$

Now from (5) and (8) it follows that

$$
2^{5} Q^{-1}<\frac{1}{2}\left|P^{\prime}(x)\right|<\left|P^{\prime}\left(\alpha_{1}\right)\right|<2\left|P^{\prime}(x)\right|<2 \delta_{0} Q .
$$

Therefore the interval $\sigma_{0}(P) \cap S_{P}\left(\alpha_{1}\right)$ is contained in $\sigma(P)$, which is the set of points $x$ in $S_{P}\left(\alpha_{1}\right) \cap I$ satisfying

$$
\left|x-\alpha_{1}\right|<6 Q^{-3}\left|P^{\prime}\left(\alpha_{1}\right)\right|^{-1} \text {. }
$$

To obtain the measure of $\widetilde{\mathcal{L}}_{3}$ it is necessary to consider five different sub-cases depending on the value of $\left|P^{\prime}\left(\alpha_{1}\right)\right|$ lying in the interval $\left(2^{5} Q^{-1}, 2 \delta_{0} Q\right)$. Throughout the proof let $v=5 / 8$.

Subcase A. Define the subset $\mathcal{L}_{31}$ of the set $\widetilde{\mathcal{L}}_{3}$, as the set of points $x \in I$, for which there exists at least one polynomial $P \in \mathcal{P}_{3}^{\prime}(Q)$, satisfying (5) and the inequality

$$
Q^{v}<\left|P^{\prime}\left(\alpha_{1}\right)\right|<2 \delta_{0} Q
$$

where $x \in S_{P}\left(\alpha_{1}\right)$.

Proposition 1. For sufficiently small $\delta_{0}$ and sufficiently large $Q$,

$$
\left|\mathcal{L}_{31}\right|<2^{-4} s|I|
$$

Proof. For a polynomial $P \in \mathcal{P}_{3}^{\prime}(Q)$ define the interval

$$
\sigma_{1}(P):=\left\{x \in S_{P}\left(\alpha_{1}\right) \cap I:\left|x-\alpha_{1}\right|<c_{6} Q^{-1}\left|P^{\prime}\left(\alpha_{1}\right)\right|^{-1}\right\}
$$

for a constant $c_{6}>1$ to be chosen later.

Using (9) and (11), it follows that

$$
|\sigma(P)|<6 c_{6}^{-1} Q^{-2}\left|\sigma_{1}(P)\right| .
$$

Note that from (10) it follows that $\left|\sigma_{1}(P)\right|<2 c_{6} Q^{-1-v}$, and for $Q>Q_{0}$ the interval $\sigma_{1}(P)$ is contained in $I$.

Now, write $P$ as a Taylor series on the interval $\sigma_{1}(P)$ so that:

$$
P(x)=P^{\prime}\left(\alpha_{1}\right)\left(x-\alpha_{1}\right)+\frac{1}{2} P^{\prime \prime}\left(\alpha_{1}\right)\left(x-\alpha_{1}\right)^{2}+\frac{1}{6} P^{\prime \prime \prime}\left(\alpha_{1}\right)\left(x-\alpha_{1}\right)^{3} .
$$

Using (10) and (11) each term is estimated to obtain

$$
|P(x)|<2 c_{6} Q^{-1}
$$

for $x \in \sigma_{1}(P)$ and $Q>Q_{0}$.

The polynomials in $P_{3}^{\prime}(Q)$ are now partitioned into sets which have the same coefficients of $x^{2}$ and $x^{3}$. For integers $a_{i}, i=2,3$, let $b_{1}$ be the pairs $\left(a_{3}, a_{2}\right)$ and let $P_{3}\left(Q, b_{1}\right)$ be the set of polynomials in $P_{3}^{\prime}(Q)$ for which the coefficient of $x^{i}$ is $a_{i}$ for $i=2$, 3 . We can assume that $a_{3}>0$ since $\sigma_{0}(P)=\sigma_{0}(-P)$. The intervals $\sigma_{1}(P)$ with $P \in P_{3}\left(Q, b_{1}\right)$ are now divided into two classes using Sprindžuk's method of essential and inessential domains [6]. First the essential intervals $\sigma_{1}(P)$ are investigated. By definition

$$
\sum_{\substack{P \in \mathcal{P}_{3}\left(Q, b_{1}\right) \\ \sigma_{1}(P) \text { essential }}}\left|\sigma_{1}(P)\right| \leqslant 2|I| .
$$


Using this, (12) and the fact that the number of different vectors $b_{1}$ does not exceed $(2 Q+1) Q$, it follows that

$$
\sum_{b_{1}} \sum_{\substack{P \in \mathcal{P}_{3}\left(Q, b_{1}\right) \\ \sigma_{1}(P) \text { essential }}}|\sigma(P)|<2^{6} c_{6}^{-1} Q^{2} Q^{-2}|I|=2^{6} c_{6}^{-1}|I| .
$$

Next, consider the inessential intervals $\sigma_{1}(P)$. For polynomials $P$ and $\bar{P}$ such that $P \neq \bar{P}$, and $P, \bar{P} \in \mathcal{P}_{3}\left(Q, b_{1}\right)$, the measure of the intersection $\sigma_{1}(P) \cap \sigma_{1}(\bar{P})=\sigma_{1}(P, \bar{P})$, exceeds $\left|\sigma_{1}(P)\right| / 2$. Hence, the inequality (13) hold for $P, \bar{P} \in \mathcal{P}_{3}\left(Q, b_{1}\right)$ and $x \in \sigma_{1}(P, \bar{P})$. As the coefficients $a_{3}$ and $a_{2}$ of the polynomials $P$ and $\bar{P}$ are the same, $R(x)=P(x)-\bar{P}(x)$ is linear and satisfies

$$
|R(x)|=|a x-b|<4 c_{6} Q^{-1}, \quad \max (|a|,|b|) \leqslant 2 Q, \quad x \in \sigma_{1}(P, \bar{P}) .
$$

Assume that $a>0$ since $\sigma^{\prime}(R)=\sigma^{\prime}(-R)$, where for the polynomial $R$ we denote by $\sigma^{\prime}(R)$ the set of points $x$ satisfying (15). The values of $a$ and $|b|$ are now estimated more precisely than in (15). In view of (6) and (10) and the fact that $x \in \sigma_{1}(P)$ it follows that $\left|P^{\prime}(x)\right|<4 \delta_{0} Q$ for $Q>Q_{0}$. Therefore $|a|=\left|P^{\prime}(x)-\bar{P}^{\prime}(x)\right|<8 \delta_{0} Q$, and using (15) we conclude that $|b|<8 \delta_{0} Q$. Thus, (15) can be rewritten as

$$
|R(x)|=|a x-b|<4 c_{6} Q^{-1}, \quad \max (a,|b|)<2^{3} \delta_{0} Q, \quad x \in \sigma_{1}(P, \bar{P}) .
$$

Now the measure of $x \in I$, for which (16) holds is estimated. For fixed $a$ and $b$ the first inequality in (16) holds for points $x \in I$ satisfying

$$
\left|x-\frac{b}{a}\right|<2^{2} c_{6} a^{-1} Q^{-1} .
$$

Denote this interval by $J(R)$, so that

$$
|J(R)|=2^{3} c_{6} a^{-1} Q^{-1} .
$$

We now wish to estimate $\sum|J(R)|$ where the sum is over $a$ and $b$, such that $b / a \in I$ and $a,|b|<2^{3} \delta_{0} Q$. For fixed $a$ denote by $M_{I}(a)$ the number of points $b$ such that these conditions hold. Then,

$$
M_{I}(a) \leqslant \begin{cases}a|I|+1 \leqslant 2 a|I| & \text { if } a \geqslant|I|^{-1}, \\ \gamma & \text { if } a<|I|^{-1},\end{cases}
$$

where $\gamma$ equals 1 or 0 . First, let $a \geqslant|I|^{-1}$, then from (18) and (19) it follows that

$$
\sum_{1 \leqslant a<8 \delta_{0} Q} \sum_{b: b / a \in I}|J(R)|<\sum_{1 \leqslant a<8 \delta_{0} Q} 2^{3} c_{6} a^{-1} Q^{-1} 2|I| a \leqslant 2^{7} c_{6} \delta_{0}|I| .
$$

Next, consider $a<|I|^{-1}$ and use the second bound in (19) to find a constant $c_{7}$ satisfying $2^{8} c_{6} s^{-1}<c_{7}<2^{-4} \delta_{0}^{-1}$ and for which the intervals

$$
J_{1}\left(R_{1}\right):=\left\{x \in I:\left|x-\frac{b_{1}}{a_{1}}\right|<c_{7} a_{1}^{-1} Q^{-1}\right\}, \quad J_{1}\left(R_{2}\right):=\left\{x \in I:\left|x-\frac{b_{2}}{a_{2}}\right|<c_{7} a_{2}^{-1} Q^{-1}\right\},
$$

where $J\left(R_{i}\right) \subseteq J_{1}\left(R_{i}\right), i=1,2$, do not intersect for $b_{1} / a_{1} \neq b_{2} / a_{2}$. To see this is possible, suppose $J_{1}\left(R_{1}\right)$ and $J_{1}\left(R_{2}\right)$ intersect nontrivially at $x$, then,

$$
\frac{1}{a_{1} a_{2}} \leqslant \frac{\left|b_{1} a_{2}-b_{2} a_{1}\right|}{a_{1} a_{2}}=\left|\frac{b_{1}}{a_{1}}-\frac{b_{2}}{a_{2}}\right| \leqslant\left|x-\frac{b_{1}}{a_{1}}\right|+\left|x-\frac{b_{2}}{a_{2}}\right| \leqslant c_{7} Q^{-1}\left(\frac{1}{a_{1}}+\frac{1}{a_{2}}\right) .
$$


Assuming WLOG that $a_{2}>a_{1}$, this gives

$$
1 \leqslant c_{7} Q^{-1}\left(a_{1}+a_{2}\right)<2 c_{7} a_{2} Q^{-1}<2^{4} c_{7} \delta_{0}
$$

which is a contradiction. Thus,

$$
\sum_{R}\left|J_{1}(R)\right|=\sum_{1 \leqslant a<8 \delta_{0} Q} 2 c_{7}^{-1} a^{-1} Q^{-1} \gamma \leqslant|I| .
$$

From this it follows that

$$
\sum_{1 \leqslant a<8 \delta_{0} Q} \gamma a^{-1} \leqslant 2^{-1} c_{7}^{-1} Q|I|
$$

For fixed $a$ and $b$ the measure of the set $x \in I$, satisfying (17), does not exceed $2^{3} c_{6} a^{-1} Q^{-1}$. Hence, summing over $b$, from the second inequality in (19) it follows that $\sum_{b: b / a \in I} \gamma a^{-1} \leqslant$ $2^{3} c_{6} a^{-1} Q^{-1} \gamma$. Using (22), it follows that

$$
\sum_{1 \leqslant a<8 \delta_{0} Q} \sum_{b: b / a \in I} 2^{3} c_{6} a^{-1} Q^{-1} \leqslant 2^{3} c_{6} Q^{-1} \sum_{1 \leqslant a<8 \delta_{0} Q} \gamma a^{-1} \leqslant 2^{2} c_{6} c_{7}^{-1}|I| \leqslant 2^{-6} s|I|
$$

if $c_{7} \geqslant 2^{8} c_{6} s^{-1}$. Therefore

$$
\left|\mathcal{L}_{31}\right|<\left(2^{6} c_{6}^{-1}+2^{7} c_{6} \delta_{0}+2^{-6} s\right)|I| .
$$

Choosing $c_{6}=2^{12} s^{-1}, \delta_{0} \leqslant 2^{-25} s^{2}$ and $c_{7}=2^{41 / 2} s^{-2}$ completes the proof.

Subcase B. Define the subset $\mathcal{L}_{32}$ of the set $\widetilde{\mathcal{L}}_{3}$, as the set of points $x \in I$, for which there exists at least one polynomial $P \in \mathcal{P}_{3}^{\prime}(Q)$, satisfying (5) and the inequality

$$
2^{8} s^{-1 / 2}<\left|P^{\prime}\left(\alpha_{1}\right)\right| \leqslant Q^{5 / 8}
$$

with $x \in S_{P}\left(\alpha_{1}\right)$.

Proposition 2. For sufficiently large $Q$,

$$
\left|\mathcal{L}_{32}\right|<2^{-4} s|I| \text {. }
$$

Proof. The proof of proposition 2 closely follows that of proposition 1, so some details will be omitted. As before, for $P \in \mathcal{P}_{3}^{\prime}(Q)$ and some positive constant $c_{9}>1$ (which will be specified later) consider the interval $\sigma(P)$ and define the interval

$$
\sigma_{2}(P):=\left\{x \in S_{P}\left(\alpha_{1}\right) \cap I:\left|x-\alpha_{1}\right|<c_{9} Q^{-1}\left|P^{\prime}\left(\alpha_{1}\right)\right|^{-1}\right\} .
$$

It is clear that

$$
|\sigma(P)|<6 c_{9}^{-1} Q^{-2}\left|\sigma_{2}(P)\right| .
$$

The definition of $\mathcal{L}_{32}$ implies that $\left|\sigma_{2}(P)\right|<|I|$. Expand $P$ and $P^{\prime}$ as Taylor series on $\sigma_{2}(P)$, to obtain

$$
|P(x)|<2 c_{9} Q^{-1}
$$

and

$$
\left|P^{\prime}(x)\right|<2\left|P^{\prime}\left(\alpha_{1}\right)\right| \text {. }
$$

Now consider the essential and inessential domains $\sigma_{2}(P)$, with $P \in \mathcal{P}_{3}\left(Q, b_{1}\right)$ where $b_{1}$ is as in proposition 1.

Following the proof of proposition 1 we obtain

$$
\sum_{b_{1}} \sum_{\substack{P \in \mathcal{P}_{3}\left(Q, b_{1}\right) \\ \sigma_{2}(P) \text { essential }}}|\sigma(P)|<2^{6} c_{9}^{-1}|I|
$$


Now the inessential domains are considered. Assume $\sigma_{2}(P), P \in P_{3}\left(Q, b_{1}\right)$, is inessential. Thus there exists $\bar{P} \in \mathcal{P}_{3}\left(Q, b_{1}\right)$ with $P \neq \bar{P}$ such that

$$
\left|\sigma_{2}(P, \bar{P})\right|=\left|\sigma_{2}(P) \cap \sigma_{2}(\bar{P})\right| \geqslant \frac{1}{2}\left|\sigma_{2}(P)\right| .
$$

Let $T(x)=P(x)-\bar{P}(x)=g x-d$, then from $(26)$ and $(27)$

$$
|g x-d|<4 c_{9} Q^{-1}, \quad 1 \leqslant g<4 Q^{5 / 8} .
$$

The inequality (29) holds on an interval $J_{2}(T)$ with centre $d / g$ and length $8 c_{9} g^{-1} Q^{-1}$. Fix $g$ and denote by $M_{I}^{\prime}(g)$ the number of points $d / g$, belonging to $I$. As in (19),

$$
M_{I}^{\prime}(g) \leqslant \begin{cases}2 g|I| & \text { if } g \geqslant|I|^{-1} \\ \gamma & \text { if } g<|I|^{-1}\end{cases}
$$

where $\gamma$ equals 1 or 0 .

Again, first consider $g \geqslant|I|^{-1}$. Then, for $Q>Q_{0}$,

$$
\sum_{1 \leqslant g<4 Q^{5 / 8}} \sum_{d: d / g \in I}\left|J_{2}(T)\right|<2^{6} c_{9} Q^{-3 / 8}|I| \leqslant 2^{-6} s|I| .
$$

Now consider $g<|I|^{-1}$. To show the sets $J_{2}(T)$ do not intersect, larger super-sets $J_{3}(T)$ defined below are shown not to intersect. Assume that for $c_{10}>4 c_{9}$ the intervals

$$
J_{3}\left(T_{1}\right):=\left\{x \in I:\left|x-\frac{d_{1}}{g_{1}}\right|<c_{10} g_{1}^{-1} Q^{-1}\right\}, \quad J_{3}\left(T_{2}\right):=\left\{x \in I:\left|x-\frac{d_{2}}{g_{2}}\right|<c_{10} g_{2}^{-1} Q^{-1}\right\},
$$

intersect nontrivially for $d_{1} / g_{1} \neq d_{2} / g_{2}$. Then, as in (21):

$$
1 \leqslant 2 c_{10}\left(g_{1}+g_{2}\right) Q^{-1}<2^{4} c_{10} Q^{-3 / 8},
$$

which is a contradiction for $Q>Q_{0}\left(c_{10}\right)$. As in (22), it therefore follows that

$$
\sum_{1 \leqslant g<4 Q^{5 / 8}} \gamma g^{-1} \leqslant 2^{-1} c_{10}^{-1} Q|I| .
$$

For fixed $g$ the measure of the set $x \in I$, satisfying (29), does not exceed $8 c_{9} g^{-1} Q^{-1}$. From (32), it follows that

$$
\sum_{1 \leqslant g<4 Q^{5 / 8}} \sum_{d: d / g \in I} 8 c_{9} g^{-1} Q^{-1} \leqslant 8 c_{9} Q^{-1} \sum_{1 \leqslant g<4 Q^{5 / 8}} \gamma g^{-1} \leqslant 4 c_{9} c_{10}^{-1}|I| \leqslant 2^{-6} s|I|
$$

for $c_{10} \geqslant 2^{8} c_{9} s^{-1}$. From this, (28), and (30) it follows that

$$
\left|\mathcal{L}_{32}\right|<\left(2^{6} c_{9}^{-1}+2^{-6} s+2^{-6} s\right)|I| .
$$

Hence, choosing $c_{9}=2^{12} s^{-1}$ and $c_{10}=2^{20} s^{-2}$, this completes the proof of proposition 2 .

Subcase C. Denote by $\mathcal{L}_{33} \subset \widetilde{\mathcal{L}}_{3}$ the set of $x \in I$, for which there exists a polynomial $P \in$ $\mathcal{P}_{3}^{\prime}(Q)$, satisfying (5) and

$$
2^{-3}<\left|P^{\prime}\left(\alpha_{1}\right)\right| \leqslant 2^{8} s^{-1 / 2}
$$

with $x \in S_{P}\left(\alpha_{1}\right)$. 
Proposition 3. For sufficiently large $Q$,

$$
\left|\mathcal{L}_{33}\right|<2^{-4} s|I| \text {. }
$$

Proof. For $P \in \mathcal{P}_{3}^{\prime}(Q)$ and some $c_{11}>1$ chosen later, define the interval

$$
\sigma_{3}(P):=\left\{x \in S_{P}\left(\alpha_{1}\right) \cap I:\left|x-\alpha_{1}\right|<c_{11} Q^{-1}\left|P^{\prime}\left(\alpha_{1}\right)\right|^{-1}\right\} .
$$

Develop $P$ and $P^{\prime}$ as a Taylor series on $\sigma_{3}(P)$, to obtain

$$
|P(x)|<2^{9} c_{11}^{2} Q^{-1}, \quad\left|P^{\prime}(x)\right|<\max \left(2^{9} s^{-1 / 2}, 2^{6} c_{11}\right)
$$

for $Q>Q_{0}$. Consider again the essential and inessential domains $\sigma_{3}(P), P \in \mathcal{P}_{3}\left(Q, b_{1}\right)$ defined as in proposition 1 . As the approach is similar to previous propositions, the calculations are omitted. In the case of the essential domains the measure is at most $2^{6} c_{11}^{-1}|I|$, and choosing $c_{11}>2^{11} s^{-1}$ gives the measure of the points lying in essential domains as $2^{-5} s|I|$.

For the inessential domains, it is necessary to estimate the measure of $x \in I$, satisfying

$$
|a x-b|<2^{10} c_{11}^{2} Q^{-1}, \quad \max (a,|b|)<2 \max \left(2^{9} s^{-1 / 2}, 2^{6} c_{11}\right) .
$$

Direct calculations show that (33) holds on a set of $x \in I$, with measure at most $c_{13} Q^{-1}$ for some constant $c_{13}>0$. Choosing $c_{5} \geqslant 2^{5} c_{13} s^{-1}$ in theorem 2 , the measure of the inessential domains is at most $2^{-5} s|I|$. Together with the result for essential domains this is enough to prove the proposition.

Subcase D. For some constant $c_{14}>0$ chosen later, denote by $\mathcal{L}_{34} \subset \widetilde{\mathcal{L}}_{3}$ the set of $x \in I$, for which there exists a polynomial $P \in \mathcal{P}_{3}^{\prime}(Q)$, satisfying (5) and

$$
c_{14} Q^{-1 / 2}<\left|P^{\prime}\left(\alpha_{1}\right)\right| \leqslant 2^{-3}
$$

where $x \in S_{P}\left(\alpha_{1}\right)$.

Proposition 4. For sufficiently large $Q$,

$$
\left|\mathcal{L}_{34}\right|<2^{-4} s|I|
$$

Proof. For $P \in \mathcal{P}_{3}^{\prime}(Q)$ and some $c_{15}>1$ define the interval

$$
\sigma_{4}(P):=\left\{x \in S_{P}\left(\alpha_{1}\right) \cap I:\left|x-\alpha_{1}\right|<c_{15} Q^{-2}\left|P^{\prime}\left(\alpha_{1}\right)\right|^{-1}\right\} .
$$

Clearly

$$
|\sigma(P)|<6 c_{15}^{-1} Q^{-1}\left|\sigma_{4}(P)\right| .
$$

Fix $a_{3}$. Let the subclass of polynomials $P \in \mathcal{P}_{3}^{\prime}(Q)$ with the same leading coefficients be denoted by $\mathcal{P}_{3}\left(Q, a_{3}\right)$. Consider again the essential and inessential domains $\sigma_{4}(P), P \in \mathcal{P}_{3}\left(Q, a_{3}\right)$.

From the definition of essential domains, it follows that

$$
\sum_{\substack{P \in \mathcal{P}_{3}\left(Q, a_{3}\right) \\ \sigma_{4}(P) \text { essential }}}\left|\sigma_{4}(P)\right| \leqslant 2|I| .
$$

Since the number of $a_{3}$ does not exceed $Q$, summing over all $a_{3}$ and using (34), gives

$$
\sum_{1 \leqslant a_{3} \leqslant Q} \sum_{\substack{P \in \mathcal{P}_{3}\left(Q, a_{3}\right) \\ \sigma_{4}(P) \text { essential }}}|\sigma(P)|<2^{4} c_{15}^{-1}|I|=2^{-5} s|I|
$$

for $c_{15}=2^{9} s^{-1}$. 
Now consider the inessential domains. From the Taylor series expansions of $P_{i}(x)$ and $P_{i}^{\prime}(x)$ on $\sigma_{4}\left(P_{i_{1}}, P_{i_{2}}\right)=\sigma_{4}\left(P_{i_{1}}\right) \cap \sigma_{4}\left(P_{i_{2}}\right), P_{i_{1}}, P_{i_{2}} \in \mathcal{P}_{3}\left(Q, a_{3}\right), P_{i_{1}} \neq P_{i_{2}}$, the upper bounds of $\left|P_{i}(x)\right|$ and $\left|P_{i}^{\prime}(x)\right|$, are

$$
\left|P_{i}(x)\right|<2 c_{15} Q^{-2},
$$

and

$$
\left|P_{i}^{\prime}(x)\right|<2\left|P^{\prime}\left(\alpha_{1}\right)\right|
$$

for $c_{14} \geqslant 2^{2} c_{15}^{1 / 2}$. Since the leading coefficients of $P_{i_{1}}$ and $P_{i_{2}}$ are equal, then the polynomial

$$
S(x)=P_{i_{1}}(x)-P_{i_{2}}(x)=f_{2} x^{2}+f_{1} x+f_{0}
$$

and by (36),

$$
|S(x)|<4 c_{15} Q^{-2}, \quad\left|S^{\prime}(x)\right|<4\left|P^{\prime}\left(\alpha_{1}\right)\right|, \quad\left|f_{i}\right| \leqslant 2 Q, \quad 0 \leqslant i \leqslant 2 .
$$

Let $\beta_{1}, \beta_{2} \in \mathbb{C}$ be the roots of $S$. Since the discrimimant $D(S)$ of $S$ satisfies

$$
|D(S)|=\left|S^{\prime}\left(\beta_{1}\right)\right|^{2}<16\left|P^{\prime}\left(\alpha_{1}\right)\right|^{2} \leqslant 2^{-2},
$$

this implies that $D(S)=0$ and that $S$ has a repeated root. Hence $S$ has the form

$$
S(x)=S_{0}^{2}(x)=\left(s_{1} x-s_{0}\right)^{2}, \quad\left|s_{1}\right|<2 Q^{1 / 2} .
$$

Thus, we need to find the measure of $x \in I$, satisfying

$$
\left|s_{1} x-s_{0}\right|<2 c_{15}^{1 / 2} Q^{-1}, \quad\left|s_{1}\right|<2 Q^{1 / 2} .
$$

Hence, $\left|x-s_{0} / s_{1}\right|<2 c_{15}^{1 / 2}\left|s_{1}\right|^{-1} Q^{-1}$, which defines an interval $J_{4}\left(S_{0}\right)$ with centre at $s_{0} / s_{1}$ and length $4 c_{15}^{1 / 2}\left|s_{1}\right|^{-1} Q^{-1}$. Fix $s_{1}$ and denote by $M_{I}^{\prime \prime}\left(s_{1}\right)$ the number of points $s_{0} / s_{1}$, belonging to $I$. As in (19), the following bounds

$$
M_{I}^{\prime \prime}\left(s_{1}\right) \leqslant \begin{cases}2 s_{1}|I| & \text { if } s_{1} \geqslant|I|^{-1} \\ \gamma & \text { if } s_{1}<|I|^{-1}\end{cases}
$$

are obtained, where $\gamma$ equals 1 or 0 .

Consider $s_{1} \geqslant|I|^{-1}$. For $Q>Q_{0}$,

$$
\sum_{1 \leqslant s_{1}<2 Q^{1 / 2}} \sum_{s_{0}: s_{0} / s_{1} \in I}\left|J_{4}\left(S_{0}\right)\right|<2^{4} c_{15}^{1 / 2} Q^{-1 / 2}|I| \leqslant 2^{-6} s|I| .
$$

Next let $s_{1}<|I|^{-1}$. If for $c_{16}>2 c_{15}^{1 / 2}$ the intervals

$$
J_{5}\left(S_{1}\right):=\left\{x \in I:\left|x-\frac{s_{0,1}}{s_{1,1}}\right|<c_{16} s_{1,1}^{-1} Q^{-1}\right\}, \quad J_{5}\left(S_{2}\right):=\left\{x \in I:\left|x-\frac{s_{0,2}}{s_{1,2}}\right|<c_{16} s_{1,2}^{-1} Q^{-1}\right\}
$$

intersect nontrivially for $s_{0,1} / s_{1,1} \neq s_{0,2} / s_{1,2}$, then as in (21):

$$
1 \leqslant 2 c_{16}\left(s_{1,1}+s_{1,2}\right) Q^{-1}<2^{3} c_{16} Q^{-1 / 2},
$$

which is a contradiction for $Q>Q_{0}\left(c_{16}\right)$. Thus, using the same arguments as in proposition 1 , as in $(22)$

$$
\sum_{1 \leqslant s_{1}<2 Q^{1 / 2}} \gamma s_{1}^{-1} \leqslant 2^{-1} c_{16}^{-1} Q|I|
$$


Since for fixed $s_{1}$ and $s_{0}$ the measure of the set $x \in I$, satisfying (38), is at most $2 c_{15}^{1 / 2}\left|s_{1}\right|^{-1} Q^{-1}$, using (41), we get

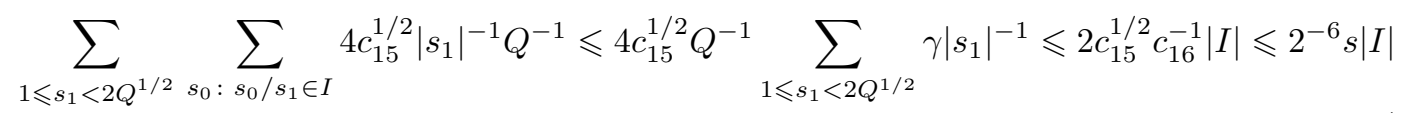

for $c_{16} \geqslant 2^{7} c_{15}^{1 / 2} s^{-1}$. Choose $c_{14}=2^{7} s^{-1 / 2}$ and $c_{16} \geqslant 2^{12} s^{-3 / 2}$. Summing the estimates (35), (39) and (42), for the measures in the essential and inessential cases, it follows that $\left|\mathcal{L}_{34}\right|<2^{-4} s|I|$. This concludes the proof of proposition 4.

Subcase E. Denote by $\mathcal{L}_{35} \subset \widetilde{\mathcal{L}}_{3}$ the set of $x \in I$, for which there exists $P \in \mathcal{P}_{3}^{\prime}(Q)$, satisfying (5) and the following condition

$$
2^{5} Q^{-1}<\left|P^{\prime}\left(\alpha_{1}\right)\right| \leqslant 2^{8} s^{-1 / 2} Q^{-1 / 2}
$$

with $x \in S_{P}\left(\alpha_{1}\right)$.

Proposition 5. For sufficiently large $Q$,

$$
\left|\mathcal{L}_{35}\right|<2^{-2} s|I| \text {. }
$$

Proof. Divide the interval $I$ into smaller intervals $J_{i}$, where $\left|J_{i}\right|=Q^{-u}$ and $u>1$. We say the polynomial $P$ belongs to the interval $J_{i}$ if there exists $x \in J_{i}$ such that (3) and (43) hold.

There is at most one irreducible polynomial belonging to an interval $J_{i}$. To see this, suppose the opposite. Assume there exists a point $x \in J_{i}$, for which (3) and (43) hold for two irreducible polynomials $P_{1}$ and $P_{2}$ from $\mathcal{P}_{3}^{\prime}(Q)$. Expand $P_{1}$ as Taylor's series on $J_{i}$ so that:

$$
P_{1}(x)=P_{1}^{\prime}\left(\alpha_{1}\right)\left(x-\alpha_{1}\right)+\frac{1}{2} P_{1}^{\prime \prime}\left(\alpha_{1}\right)\left(x-\alpha_{1}\right)^{2}+\frac{1}{6} P_{1}^{\prime \prime \prime}\left(\alpha_{1}\right)\left(x-\alpha_{1}\right)^{3} .
$$

Using the estimate for $\left|P_{1}^{\prime}\left(\alpha_{1}\right)\right|$ from (43), and the trivial bounds $\left|P^{(k)}\left(\alpha_{1}\right)\right| \ll Q, k=2,3$, we obtain

$$
\left|P_{1}(x)\right|<2^{9} s^{-1 / 2} Q^{-1 / 2-u}, \quad x \in J_{i},
$$

for $u>3 / 2$. Obviously the same estimate holds for $P_{2}$ in $J_{i}$.

We use the following lemma which is proved in [7].

Lemma 2. Let $\delta>0$ and $Q>Q_{0}(\delta)$. Further, let $P_{1}$ and $P_{2}$ be two integer polynomials of degree at most $n$ with no common roots, and $\max \left(H\left(P_{1}\right), H\left(P_{2}\right)\right) \leqslant Q$. Let $J \subset \mathbb{R}$ be an interval of length $|J|=Q^{-\eta}, \eta>0$. If there exists $\tau>0$, such that for all $x \in J$

$$
\left|P_{j}(x)\right|<Q^{-\tau},
$$

for $j=1,2$, then

$$
\tau+1+2 \max (\tau+1-\eta, 0)<2 n+\delta .
$$

Applying lemma 2 with $\tau=1 / 2+u-\epsilon, \epsilon>0$, and $\eta=u$, leads to a contradiction in (44) if $u>3 / 2+\delta+3 \epsilon$. Choose $u$ satisfying $3 / 2+\delta+3 \epsilon<u<2$ and it follows that there is at most one irreducible polynomial on any $J_{i}$. As there is at most one polynomial $P \in \mathcal{P}_{3}^{\prime}(Q)$ that belongs to any $J_{i}$ then by Lemma 1 , the measure of those $x$, satisfying (3) and (43), does not exceed

$$
2^{-3} Q^{-2+u}|I|<2^{-4} s|I|
$$

for $u<2$ and $Q>Q_{0}$.

If $P \in \mathcal{P}_{3}^{\prime}(Q)$ is a reducible polynomial belonging to $J_{i}$ then $P(x)=t_{1}(x) t_{2}(x)$, where $t_{1}$ is a polynomial of degree one and $t_{2}$ is either an irreducible polynomial or degree two or the product 
of two linear polynomials. Let $t_{1}(x)=a x+b$ and $t_{2}(x)=b_{2} x^{2}+b_{1} x+b_{0}$. Assume that $a>0$. By Gelfond's Lemma [4],

$$
a H\left(t_{2}\right) \leqslant H\left(t_{1}\right) H\left(t_{2}\right) \leqslant 2^{3} H(P) \leqslant 2^{3} Q .
$$

Denote by $\mathcal{L}_{351}$ the set of $x \in I$, for which the system

$$
\left|t_{1}(x)\right|<Q^{-1}, \quad a<\delta_{0} Q
$$

is satisfied by some polynomial $t_{1}$. The system (46) is similar to (16). Hence, using the same arguments, it can be shown that $\left|\mathcal{L}_{351}\right|<2^{-5} s|I|$. If (46) does not hold, there are three possibilities:

$$
\begin{array}{rrr}
\left|t_{1}(x)\right|<Q^{-1}, & \delta_{0} Q \leqslant a & <2^{3} Q, \\
\left|t_{1}(x)\right| \geqslant Q^{-1}, & a<\delta_{0} Q, \\
\left|t_{1}(x)\right| \geqslant Q^{-1}, & \delta_{0} Q \leqslant a & <2^{3} Q .
\end{array}
$$

For (47) there are two further possibilities; namely,

$$
\delta_{0} Q^{-1}<\left|t_{1}(x)\right|<Q^{-1}, \quad \delta_{0} Q \leqslant a<2^{3} Q
$$

and

$$
\left|t_{1}(x)\right| \leqslant \delta_{0} Q^{-1}, \quad \delta_{0} Q \leqslant a<2^{3} Q .
$$

Each of these will be considered in turn. Denote by $\mathcal{L}_{352}$ and by $\mathcal{L}_{353}$ the sets of $x \in I$, for which (50) and (51) are satisfied for polynomials $t_{1}$ respectively. The system (51) is similar to (16), and it is not difficult to show that $\left|\mathcal{L}_{353}\right|<2^{-5} s|I|$.

Turning to $\mathcal{L}_{352}$ and using (45), (50) and $|P(x)|<Q^{-3}$ it follows that

$$
\left|t_{2}(x)\right| \leqslant \delta_{0}^{-1} Q^{-2}, \quad H\left(t_{2}\right)<2^{3} \delta_{0}^{-1} .
$$

The number of polynomials that satisfy the second inequality in (52) does not exceed a constant depending on $\delta_{0}$, say $c\left(\delta_{0}\right)$, therefore we conclude that $\left|\mathcal{L}_{352}\right|<2^{-5} s|I|$.

Now we consider (48). Using (48) and (45)

$$
\left|t_{2}(x)\right|<Q^{-2}, \quad H\left(t_{2}\right)<2^{3} Q .
$$

First, $t_{2}^{\prime}$ is estimated from above on $J_{i}$. Using the equations

$$
P^{\prime}(x)=t_{1}^{\prime}(x) t_{2}(x)+t_{1}(x) t_{2}^{\prime}(x),
$$

and

$$
P^{\prime}(x)=P^{\prime}\left(\alpha_{1}\right)+P^{\prime \prime}\left(\alpha_{1}\right)\left(x-\alpha_{1}\right)+P^{\prime \prime \prime}\left(\alpha_{1}\right) \frac{\left(x-\alpha_{1}\right)^{2}}{2},
$$

the estimates (48), (53) and

$$
\left|P^{\prime}(x)\right|<2^{9} s^{-1 / 2} Q^{-1 / 2}, \quad Q^{-1} \leqslant\left|t_{1}(x)\right| \ll Q,
$$

gives a contradiction for $\left|t_{2}^{\prime}(x)\right|>Q^{5 / 8}$ for sufficiently large $Q$. Thus, $\left|t_{2}^{\prime}(x)\right| \leqslant Q^{5 / 8}, x \in J_{i}$. Then from (53),

$$
\left|t_{2}(x)\right|<Q^{-2}, \quad\left|t_{2}^{\prime}(x)\right| \leqslant Q^{5 / 8}
$$

hold. Denote by $\mathcal{L}_{354}$ the set of $x \in I$, for which (54) is satisfied for a polynomial $t_{2}$. The measure of $\mathcal{L}_{354}$ is estimated in a manner similar to proposition 2 , giving $\left|\mathcal{L}_{354}\right|<2^{-4} s|I|$.

Finally, we consider (49). Using (45), (49) and $|P(x)|<Q^{-3}$, it follows that

$$
\left|t_{2}(x)\right|<Q^{-2}, \quad H\left(t_{2}\right)<2^{3} \delta_{0}^{-1} \text {. }
$$


Denote by $\mathcal{L}_{355}$ the set of $x \in I$, for which (55) is satisfied for some $t_{2}$. The number of polynomials which satisfy the second inequality in $(55)$ is bounded by a constant $c_{2}\left(\delta_{0}\right)$, therefore we conclude that $\left|\mathcal{L}_{355}\right|<2^{-5} s|I|$.

Thus,

$$
\left|\mathcal{L}_{35}\right|<2^{-4} s|I|+\sum_{i=1}^{5}\left|\mathcal{L}_{35 i}\right|<2^{-2} s|I|,
$$

which completes the proof of the proposition.

Case 2. Define the subset $\check{\mathcal{L}}_{3}$ of the set $\overline{\mathcal{L}}_{3}$, as the set of points $x \in I$, for which there exists at least one polynomial $P \in \mathcal{P}_{3}^{\prime}(Q)$ such that

$$
|P(x)|<Q^{-3}, \quad\left|P^{\prime}(x)\right| \leqslant 2^{6} Q^{-1} .
$$

Define by $\sigma_{*}(P)$ the set of solutions to (56) for a fixed polynomial $P \in \mathcal{P}_{3}^{\prime}(Q)$. Let $x \in \sigma_{*}(P) \cap$ $S_{P}\left(\alpha_{1}\right)$. First, it is shown that the value of the derivative of $P$ at $\alpha_{1}, P\left(\alpha_{1}\right)=0$, satisfies

$$
\left|P^{\prime}\left(\alpha_{1}\right)\right| \leqslant 2^{8} Q^{-1}
$$

To show this, assume the opposite holds. Develop $P^{\prime}$ as a Taylor series in the neighborhood of $\alpha_{1}$, to obtain

$$
P^{\prime}(x)=P^{\prime}\left(\alpha_{1}\right)+P^{\prime \prime}\left(\alpha_{1}\right)\left(x-\alpha_{1}\right)+\frac{1}{2} P^{\prime \prime \prime}\left(\alpha_{1}\right)\left(x-\alpha_{1}\right)^{2},
$$

where $\left|x-\alpha_{1}\right|<2^{-6} Q^{-2}$ by lemma 1 . Since $\left|P^{k}\left(\alpha_{1}\right)\right| \ll Q$ for $k=1,2,3$, it follows that

$$
\max \left(\left|P^{\prime \prime}\left(\alpha_{1}\right)\left(x-\alpha_{1}\right)\right|,\left|\frac{1}{2} P^{\prime \prime \prime}\left(\alpha_{1}\right)\left(x-\alpha_{1}\right)^{2}\right|\right)<2^{-3} Q^{-1},
$$

and hence $\left|P^{\prime}(x)\right|>2^{7} Q^{-1}$, which contradicts the condition that $\left|P^{\prime}(x)\right| \leqslant 2^{6} Q^{-1}$.

To estimate the measure of $\breve{\mathcal{L}}_{3}$ two cases, depending on the value of $\left|P^{\prime}\left(\alpha_{1}\right)\right|$, need to be considered. For some constant $0<c_{17}<1 / 6$ denote by $\mathcal{L}_{36} \subset \breve{\mathcal{L}}_{3}$ the set of $x \in I$, for which there exists a polynomial $P \in \mathcal{P}_{3}^{\prime}(Q)$, satisfying (56) and

$$
\left|P^{\prime}\left(\alpha_{1}\right)\right|<c_{17} Q^{-1}
$$

with $x \in S_{P}\left(\alpha_{1}\right)$.

Proposition 6. For sufficiently large $Q$,

$$
\left|\mathcal{L}_{36}\right|<2^{-2} s|I|
$$

Proof. First note that

$$
1 \leqslant|D(P)|=\left|a_{3}^{4}\left(\alpha_{1}-\alpha_{2}\right)^{2}\left(\alpha_{1}-\alpha_{3}\right)^{2}\left(\alpha_{2}-\alpha_{3}\right)^{2}\right|=\left|P^{\prime}\left(\alpha_{1}\right)\right|^{2} a_{3}^{2}\left|\alpha_{2}-\alpha_{3}\right|^{2} .
$$

Also, $\left|\alpha_{2}-\alpha_{3}\right| \leqslant 6 Q /\left|a_{3}\right|$ as $\left|\alpha_{i}\right| \leqslant 3 Q /\left|a_{3}\right|, i=2,3$. Finally, as $\left|P^{\prime}\left(\alpha_{1}\right)\right|<c_{17} Q^{-1}$, this gives

$$
1 \leqslant D(P) \leqslant 2^{2} \cdot 3^{2} c_{17}^{2} Q^{-2} Q^{2}=2^{2} \cdot 3^{2} c_{17}^{2},
$$

which does not hold when $c_{17}<1 / 6$ thus, the discriminant of $P$ satisfies $D(P)=0$, which implies that $P$ has a repeated root. Following the same approach as in proposition 4 , it follows that $\left|\mathcal{L}_{36}\right|<2^{-2} s|I|$. 
The second sub-case of case 2 is now considered. Denote by $\mathcal{L}_{37} \subset \check{\mathcal{L}}_{3}$ the set of $x \in I$, for which there exists some polynomial $P \in \mathcal{P}_{3}^{\prime}(Q)$, satisfying (56) and

$$
c_{17} Q^{-1} \leqslant\left|P^{\prime}\left(\alpha_{1}\right)\right| \leqslant 2^{8} Q^{-1}
$$

with $x \in S_{P}\left(\alpha_{1}\right)$.

Proposition 7. For Q sufficiently large,

$$
\left|\mathcal{L}_{37}\right|<2^{-2} s|I|
$$

Proof. Divide the interval $I$ into smaller intervals $J_{i}^{\prime}$ with $\left|J_{i}^{\prime}\right|=Q^{-u^{\prime}}$, where $u^{\prime}>3 / 2$. The assumption that at least two irreducible polynomials belong to the interval $J_{i}^{\prime}$ will lead to a contradiction. To show this, suppose that $P_{1}$ and $P_{2}$ both belong to $J_{i}^{\prime}$. Expand $P_{1}$ as Taylor series on $J_{i}^{\prime}$, to obtain:

$$
\left|P_{1}(x)\right|<2^{5} Q^{1-2 u^{\prime}}, \quad x \in J_{i}^{\prime},
$$

for $u^{\prime}<2$. Obviously the same estimate holds for $P_{2}$ on $J_{i}^{\prime}$. Applying Lemma 2 with $\tau=-1+$ $2 u^{\prime}-\epsilon^{\prime}, \epsilon^{\prime}>0$, and $\eta=u^{\prime}$, leads to a contradiction in (44) for $u^{\prime}>3 / 2+\delta / 4+3 \epsilon^{\prime} / 4$. Thus, choose $u^{\prime}$, satisfying $3 / 2+\delta / 4+3 \epsilon^{\prime} / 4<u^{\prime}<2$.

Hence there is at most one polynomial $P \in \mathcal{P}_{3}^{\prime}(Q)$ belonging to each $J_{i}^{\prime}$. Therefore, by lemma 1 the measure of those $x$, satisfying the first inequality of (3) and (58), is at most

$$
4 c_{17}^{-1} Q^{-2+u^{\prime}}|I|<2^{-4} s|I|
$$

for $u^{\prime}<2$ and $Q>Q_{0}$.

When the polynomials are reducible, the proof exactly follows proposition 5.

Adding the measures calculated in propositions 1 to 7 , it follows that the measure of $\overline{\mathcal{L}}_{3}$ satisfies (4).

Finally, we turn to the proof of Theorem 1.

\section{Proof of Theorem 1}

For the rest of the proof we may assume that $c_{5}$ is a constant which is greater or equal to $2 \cdot 3^{5} /(1-s) \delta_{0}$ and for which Theorem 2 is valid.

Denote by $\mathcal{L}_{0}(Q, I)$ the set of $x \in I$, for which the inequality $|P(x)|<Q^{-3}$ is satisfied for some $P \in \mathcal{P}_{3}^{\prime}(Q)$. It can be readily verified using Dirichlet's box principle that $\mathcal{L}_{0}(Q, I)=I$. By theorem 2 there exists a set $\mathcal{L}_{3}\left(Q, \delta_{0}, I\right)=I \backslash \overline{\mathcal{L}}_{3}\left(Q, \delta_{0}, I\right) \subset I$ such that $\left|\mathcal{L}_{3}\left(Q, \delta_{0}, I\right)\right| \geqslant(1-s)|I|$ for all $Q>Q_{0}$, where $Q_{0}>c_{5}|I|^{-1}$.

Denote by $\mathcal{L}_{\leqslant 2}\left(Q, \delta_{0}, I\right)$ the union of the intervals $\sigma(\alpha)=\left\{x \in I:|x-\alpha|<3 \delta_{0}^{-1} Q^{-4}\right\}$ over all real algebraic numbers of degree at most 2 and height at most $Q$. The number of different intervals in this union is at most $(2 Q+1)^{3}$ and every interval has a length at most $6 \delta_{0}^{-1} Q^{-4}$, therefore we conclude that $\left|\mathcal{L}_{\leqslant 2}\left(Q, \delta_{0}, I\right)\right| \leqslant(1-s)|I| / 2$ for $c_{5} \geqslant 2 \cdot 3^{5} /(1-s) \delta_{0}$.

Let $\mathcal{L}_{3}^{\prime}\left(Q, \delta_{0}, I\right)$ be defined by $\mathcal{L}_{3}^{\prime}\left(Q, \delta_{0}, I\right)=\mathcal{L}_{3}\left(Q, \delta_{0}, I\right) \backslash \mathcal{L}_{\leqslant 2}\left(Q, \delta_{0}, I\right)$. Let $x \in \mathcal{L}_{3}^{\prime}\left(Q, \delta_{0}, I\right)$. Then there exists a non-zero polynomial $P \in \mathcal{P}_{3}^{\prime}(Q)$, satisfying

$$
|P(x)|<Q^{-3}, \quad\left|P^{\prime}(x)\right| \geqslant \delta_{0} Q .
$$

It will be shown that there exists a root $\alpha$ of $P$ close to $x$. Let $y \in \mathbb{R}$, be such that $|y-x|=$ $3 \delta_{0}^{-1} Q^{-4}$. By Taylor's formula

$$
P(y)=\sum_{i=0}^{3} \frac{1}{i !} P^{(i)}(x)(y-x)^{i} .
$$


As $x \in[-1 / 2,1 / 2],\left|P^{(i)}(x)\right| \ll Q$ for $i=1,2,3$. It is readily verified that

$$
\left|P^{(i)}(x)(y-x)^{i}\right| \ll Q^{-7} \quad \text { for } \quad i \geqslant 2 .
$$

Also, by (59), $|P(x)|<Q^{-3}$. Thus,

$$
\sum_{i=0,2,3}\left|\frac{1}{i !} P^{(i)}(x)(y-x)^{i}\right|<Q^{-3}+\sum_{i=2}^{3} 3^{2}\left(7 \delta_{0}^{-2} Q^{-7}\right)<2 Q^{-3} .
$$

On the other hand, by (59)

$$
\left|P^{\prime}(x)(y-x)\right| \geqslant 3 Q^{-3} \text {. }
$$

By (60) and (61) the behaviour of $P(y)$ is dominated by the behaviour of $P^{\prime}(x)(y-x)$. It also follows from $(60)$ and $(61)$ that $P(y)$ has different signs at the endpoints of the interval $\left(x-3 \delta_{0}^{-1} Q^{-4}\right.$, $\left.x+3 \delta_{0}^{-1} Q^{-4}\right)$. Thus, by the continuity of $P$, there exists a root $\alpha$ of $P$ in this interval, and

$$
|x-\alpha|<3 \delta_{0}^{-1} Q^{-4}
$$

Since $x \notin \mathcal{L}_{\leqslant 2}\left(Q, \delta_{0}, I\right)$ then we conclude that the degree of $\alpha$ is exactly 3 .

Choose a maximal collection of real algebraic numbers $\left\{\alpha_{1}, \ldots, \alpha_{t}\right\} \subset I$, with degree $\operatorname{deg} \alpha_{i}=3$ satisfying

$$
H\left(\alpha_{i}\right) \leqslant Q, \quad\left|\alpha_{i}-\alpha_{j}\right| \geqslant 3 \delta_{0}^{-1} Q^{-4}, \quad 1 \leqslant i<j \leqslant t .
$$

As has been shown, for any $x \in \mathcal{L}_{3}^{\prime}\left(Q, \delta_{0}, I\right)$ there exists $\alpha \in A_{3}$, satisfying (62) with $H(\alpha) \leqslant Q$. Since the collection $\left\{\alpha_{1}, \ldots, \alpha_{t}\right\}$ is maximal, there exists $\alpha_{i}$ in this collection such that $\left|\alpha-\alpha_{i}\right| \leqslant$ $3 \delta_{0}^{-1} Q^{-4}$. From this and (62), by the triangle inequality it follows that $\left|x-\alpha_{i}\right|<6 \delta_{0}^{-1} Q^{-4}$. Then

$$
\mathcal{L}_{3}^{\prime}\left(Q, \delta_{0}, I\right) \subset \bigcup_{i=1}^{t}\left\{x \in I:\left|x-\alpha_{i}\right|<6 \delta_{0}^{-1} Q^{-4}\right\} .
$$

Using $\left|\mathcal{L}_{3}^{\prime}\left(Q, \delta_{0}, I\right)\right| \geqslant(1-s)|I| / 2$, this gives

$$
t \geqslant 2^{-3} 3^{-1} \delta_{0}(1-s) Q^{4}|I|
$$

Let $T=Q^{4}$, then for any $T \geqslant T_{0}$, where

$$
T_{0}=\left(c_{5}+1\right)^{4}|I|^{-4},
$$

there exists a collection $\alpha_{1}, \ldots, \alpha_{t} \in I \cap A_{3}$ satisfying (2) which completes the proof of the theorem.

The authors are grateful to the anonymous referee for the very useful comments on this paper.

\section{Bibliography}

[1] A. Baker, W. M. Schmidt, "Diophantine approximation and Hausdorff dimension", Proc. Lond. Math. Soc. (3), 21 (1970), 1-11.

[2] В.И. Берник, "О точном порядке приближения нуля значениями целочисленных многочленов", Acta Arith., 53:1 (1989), 17-28.

[3] V. Beresnevich, "On approximation of real numbers by real algebraic numbers", Acta Arith., 90:2 (1999), 97-112.

[4] Y. Bugeaud, Approximation by Algebraic Numbers, Cambridge Tracts in Math., 160, Cambridge Univ. Press, Cambridge, 2004. 
[5] В.В.Бересневич, "Эффективные оценки мер множеств действительных чисел с заданным порядком аппроксимации квадратичными иррациональностями", Изв. АН Беларуси. Сер. физ.-матем. наук, 1996, № 4, 10-15.

[6] В. Г. Спринджук, Проблема Малера в метрической теории чисел, Наука и техника, Минск, 1967; V. G. Sprindžuk, Mahler's problem in the metric theory of numbers, Trans. Math. Monogr., 25, Amer. Math. Soc., Providence, RI, 1969.

[7] В. И. Берник, "Применение размерностн Хаусдорфа в теории диофантовых приближений", Acta Arith., 42 (1983), 219-253; V. I. Bernik, "Application of Hausdorff dimension in the theory of Diophantine approximations", Amer. Math. Soc. Transl. Ser. 2, 140, Amer. Math. Soc., Providence, RI, 1988, 15-44. 